TRANSACTIONS OF THE

AMERICAN MATHEMATICAL SOCIETY

Volume 360, Number 7, July 2008, Pages 3631-3644

S 0002-9947(07)04411-X

Article electronically published on December 20, 2007

\title{
ON A RAMANUJAN EQUATION CONNECTED WITH THE MEDIAN OF THE GAMMA DISTRIBUTION
}

\author{
J. A. ADELL AND P. JODRÁ
}

Abstract. In this paper, we consider the sequence $\left(\theta_{n}\right)_{n \geq 0}$ solving the Ramanujan equation

$$
\frac{e^{n}}{2}=\sum_{k=0}^{n} \frac{n^{k}}{k !}+\frac{n^{n}}{n !}\left(\theta_{n}-1\right), \quad n=0,1, \ldots
$$

The three main achievements are the following. We introduce a continuoustime extension $\theta(t)$ of $\theta_{n}$ and show its close connections with the medians $\lambda_{n}$ of the $\Gamma(n+1,1)$ distributions and the Charlier polynomials. We give upper and lower bounds for both $\theta(t)$ and $\lambda_{n}$, in particular for $\theta_{n}$, which are sharper than other known estimates. Finally, we show (and at the same time complete) two conjectures by Chen and Rubin referring to the sequence of medians $\left(\lambda_{n}\right)_{n \geq 1}$.

\section{INTRODUCTION}

A famous problem posed by Ramanujan 25] in 1911 as Question 294 reads as follows. For any $n=0,1, \ldots$, show that

$$
\frac{e^{n}}{2}=\sum_{k=0}^{n} \frac{n^{k}}{k !}+\frac{n^{n}}{n !}\left(\theta_{n}-1\right), \quad \text { where } \quad \frac{1}{2} \geq \theta_{n} \geq \frac{1}{3}
$$

Throughout the last century, this problem has attracted a great deal of attention. Although a solution was outlined by Ramanujan himself [26, the first rigorous proofs of (1.1) and of the fact that $\theta_{n}$ decreases as $n$ increases were published independently by Szegö 29] and Watson [31. Other proofs were provided by Cheng [13, Karamata [21] and Bracken [11. Copson [16] considered a similar result in connection with $e^{-n}$, and Paris 24] extended statement (1.1) to confluent hypergeometric functions. Asymptotic expansions of $\theta_{n}$ have been obtained by many authors. For instance, Marsaglia 23 developed an efficient method to obtain an arbitrary number of terms in the asymptotic expansion of $\theta_{n}$, the first seven being

$$
\theta_{n}=u_{n}+O\left(\frac{1}{n^{7}}\right)
$$

Received by the editors November 27, 2005 and, in revised form, April 27, 2006.

2000 Mathematics Subject Classification. Primary 41A60; Secondary 60E05.

Key words and phrases. Central limit theorem, Charlier polynomials, forward difference, gamma distribution, median, Poisson process, Ramanujan's equation.

This work was supported by research projects BFM2002-04163-C02-01 and DGA E-12/25, and by FEDER funds.

(C)2007 American Mathematical Society Reverts to public domain 28 years from publication 
where

$$
\begin{aligned}
u_{n}:= & \frac{1}{3}+\frac{4}{135 n}-\frac{8}{2835 n^{2}}-\frac{16}{8505 n^{3}} \\
& +\frac{8992}{12629925 n^{4}}+\frac{334144}{492567075 n^{5}}-\frac{698752}{1477701225 n^{6}} .
\end{aligned}
$$

In his first letter to Hardy in 1913, Ramanujan [27] posed the more delicate question

$$
\theta_{n}=\frac{1}{3}+\frac{4}{135\left(n+k_{n}\right)}, \quad \text { where } \quad \frac{8}{45} \geq k_{n} \geq \frac{2}{21} .
$$

This 'ultimately famous' ([10, p. 46]) Ramanujan conjecture has waited almost a century to be completely proved. As far as we know, Choi [15] was the first to give specific lower and upper bounds for $\theta_{n}$ and, one year later, Flajolet et al. [18] provided a definitive proof of (1.4). Interesting refinements of (1.4) can be found in Alm [5], who shows that $k_{n}$ decreases as $n$ increases and in Alzer [6], who establishes that

$$
\theta_{n}=\frac{1}{3}+\frac{4}{135 n}-\frac{8}{2835\left(n+k_{n}^{*}\right)^{2}}
$$

where

$$
-\frac{1}{3}<k_{n}^{*} \leq-1+\frac{4}{\sqrt{21(368-135 e)}}
$$

the bounds for $k_{n}^{*}$ being sharp. We refer the reader to Berndt [9] and Berndt et al. [10] for historical notes, further comments and a full account of references.

Starting from Copson [16], different authors have noticed that Ramanujan's equation (1.1) has close connections to probability theory (see, for instance, Flajolet et al. [18, Bracken [1], Berndt et al. [10] and Alzer [7]). In fact, it is possible to derive asymptotic expansions of $\theta_{n}$ by using probabilistic tools, such as certain formulae involving the standard normal distribution function (Marsaglia 23]) or the central limit theorem for Poisson random variables (Cheng [13]) and gamma random variables (Key [22]).

From our point of view, however, the most interesting connection is that relating $\theta_{n}$ with the median $\lambda_{n}$ of the $\Gamma(n+1,1)$ distribution, as pointed out by Choi $[15]$ (see also Alm [5] and Alzer [7]). Two conjectures by Chen and Rubin [12 referring to $\lambda_{n}$ are certainly among the main motivations of Choi's paper.

To be more precise, let $X$ be a random variable with distribution function $F(x):=P(X \leq x), x \in \mathbb{R}$. The median of $X$ is defined as

$$
\operatorname{Med}(X):=\inf \{x \in \mathbb{R}: F(x) \geq 1 / 2\} .
$$

On the other hand, for any $n=0,1, \ldots$, let $X_{n}$ be a nonnegative random variable having the $\Gamma(n+1,1)$ distribution, that is,

$$
F_{n}(x):=P\left(X_{n} \leq x\right)=\frac{1}{n !} \int_{0}^{x} u^{n} e^{-u} d u, \quad x \geq 0 .
$$

The median $\lambda_{n}$ of $X_{n}$ is thus the unique solution to the equation $F_{n}\left(\lambda_{n}\right)=1 / 2$. Observe that the only explicit solution is $\lambda_{0}=\log 2$. In this context, conjecture 1 by Chen and Rubin [12], translated in terms of $\lambda_{n}$, says that

$$
\log 2 \geq \lambda_{n}-n>\frac{2}{3}, \quad n=0,1, \ldots,
$$


while conjecture 2 states that $\left(\lambda_{n}-n\right)_{n \geq 0}$ is a decreasing sequence. Together with a finite expansion, Choi [15] gives the asymptotic expansion

$$
\theta_{n}-1=\sum_{k=0}^{\infty} c_{k}(n)\left(n-\lambda_{n}\right)^{k+1}, \quad n=1,2, \ldots,
$$

where the coefficients $\left(c_{k}(n)\right)_{k>0}$ satisfy appropriate recurrence relations. Formula (1.9) allows Choi to prove conjecture 1 by Chen and Rubin. Conjecture 2 has been shown by Alm [5] for the first time. A short proof of both conjectures can be found in Adell and Jodrá 11. Some improvements and extensions have been obtained in two recent papers. Alzer [7] determines all real parameters $\alpha$ and $\beta$ such that $\left(\lambda_{n}-\alpha n\right)_{n \geq 0}$ is strictly decreasing and $\left(\lambda_{n}-\beta n\right)_{n \geq 0}$ is strictly increasing, whereas Berg and Pedersen 8 show that Chen and Rubin's conjectures hold for the median $\lambda(t)$ of the $\Gamma(t+1,1)$ distribution, $t>-1$.

The purpose of this paper is threefold. In the first place, we extend Ramanujan's equation (1.1) to a continuous setting. In this respect, we mention that Watson [31] and Karamata 21] (see also Berg and Pedersen [8]) considered the extension

$$
\theta_{t}=1+\frac{t}{2}\left(\int_{0}^{1}\left(u e^{1-u}\right)^{t} d u-\int_{1}^{\infty}\left(u e^{1-u}\right)^{t} d u\right), \quad t>0
$$

Although this smooth extension seems fairly natural, because any $\theta_{n}$ satisfies (1.10), numerical computations show that $\theta_{t}$ does not fulfill the equation

$$
\frac{e^{t}}{2}=\sum_{k=0}^{\lfloor t\rfloor} \frac{t^{k}}{k !}+\frac{t^{\lfloor t\rfloor}}{\lfloor t\rfloor !}\left(\theta_{t}-1\right), \quad t>0,
$$

when $t \neq 1,2, \ldots$ Here, the symbol $\lfloor\cdot\rfloor$ means an integer part. In contrast, Choi's equation (1.9) suggests that any extension $\widetilde{\theta}_{t}$ of $\theta_{n}$ should verify that $\widetilde{\theta}_{\lambda_{n}}=1$ for any $n=0,1, \ldots$ This is the main reason why we consider the left-continuous extension $\theta(t)$, having jumps at each median $\lambda_{n}$ (see Figure 10), defined by

$$
\frac{e^{t}}{2}=\sum_{k=0}^{n} \frac{t^{k}}{k !}+\frac{t^{n}}{n !}(\theta(t)-1), \quad \lambda_{n-1}<t \leq \lambda_{n}, \quad n=1,2, \ldots
$$

As we shall see in the following sections, such an extension is very natural from a probabilistic viewpoint. For the sake of simplicity, we shall always assume that $t \geq 1$ and therefore that $\theta(t)$ is defined in $[1, \infty)$.

In the second place, we give lower and upper bounds both for $\theta(t)$ and $\lambda_{n}$, in particular for $\theta_{n}$, which are sharper than other known estimates, and show that both problems are actually equivalent. In turn, the estimates for $\lambda_{n}$ are achieved by checking the monotonicity of certain sequences involving tail probabilities of adequate Poisson distributions. Such monotonicity properties are related with the problem of monotonic convergence in the central limit theorem (see the comments preceding Lemma 3.2 in Section (3). Finally, we show, and at the same time improve, the two aforementioned conjectures by Chen and Rubin.

We use a probabilistic approach in the spirit of Choi [15] by adding, as a basic ingredient, the orthogonal polynomials with respect to the Poisson distribution, namely, the Charlier polynomials. We believe that this probabilistic perspective provides simple unified proofs. It is also worth mentioning that some of the proofs in this paper rely upon exact computations of certain finite sums. We emphasize that these sums may involve dozens or even hundreds of terms, depending on the 


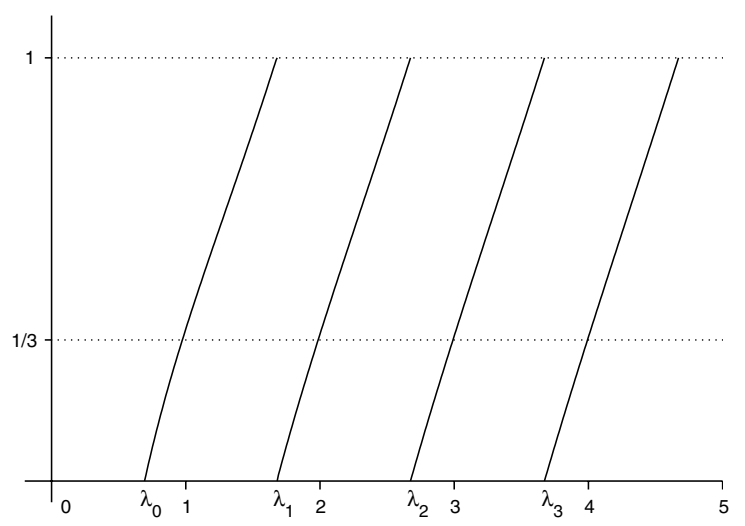

Figure 1 . The function $\theta(t), t>\lambda_{0}=\log 2$.

degree of accuracy we are working with. Therefore, the help of symbolic calculus programs to perform such sums seems to be necessary (see Remark 3.3 at the end of Section 31). Perhaps this explains why Ramanujan's conjecture (1.4) has waited almost a century to be completely proved.

The main results, together with a comparative discussion with well established results in the literature, are stated in Section 2, whereas their proofs are postponed to Section 3. Some remarks on further improvements and certain open questions concerning the complete monotonicity of the sequence $\left(\lambda_{n}-n\right)_{n \geq 1}$ close the paper.

\section{MAIN RESULTS}

Denote by $\mathbb{N}$ the set of nonnegative integers and by $\mathbb{N}^{*}:=\mathbb{N} \backslash\{0\}$. Denote also by $f_{+}(s)$ the right limit of the function $f$ at $s$. Although it is not explicitly written from now on, recall that the continuous variable $t$ is always assumed to be not less than 1. We first consider some properties of the extension $\theta(t)$ defined in (1.11). In accordance with Figure 1, the following proposition shows that $\theta(t)$ is almost a straight line from 0 to 1 between two consecutive medians.

Proposition 2.1. For any $n \in \mathbb{N}^{*}$ and $\lambda_{n-1}<t<\lambda_{n}$, we have

(i) $0=\theta_{+}\left(\lambda_{n-1}\right)<\theta(t)<\theta\left(\lambda_{n}\right)=1$.

(ii) $0<t \theta^{\prime}(t)=(t-n) \theta(t)+n$.

Recall that the standard Poisson process $\left(N_{s}\right)_{s \geq 0}$ is a process with independent stationary increments, right-continuous nondecreasing paths, and such that $N_{0}=0$ and

$$
P\left(N_{s}=n\right):=e^{-s} \frac{s^{n}}{n !}, \quad n \in \mathbb{N}, \quad s>0 .
$$

Let $\left(C_{k}(t ; x)\right)_{k \geq 0}$ be the sequence of Charlier polynomials with respect to $N_{t}$. These orthogonal polynomials may be defined in various equivalent ways (see Chihara 14 , Chap. VI]). For instance, by the generating function

$$
e^{-t w}(1+w)^{x}=\sum_{k=0}^{\infty} C_{k}(t ; x) \frac{(-t w)^{k}}{k !},
$$


by the three-term recurrence relation

$$
-t C_{k+1}(t ; x)=(x-k-t) C_{k}(t ; x)+k C_{k-1}(t ; x), \quad k \in \mathbb{N},
$$

with initial conditions $C_{-1}(t ; x)=0$ and $C_{0}(t ; x)=1$, or, explicitly, by

$$
C_{k}(t ; x):=\sum_{i=0}^{k}\left(\begin{array}{l}
k \\
i
\end{array}\right)\left(\begin{array}{l}
x \\
i
\end{array}\right) i !(-t)^{-i}, \quad k \in \mathbb{N} .
$$

The Charlier polynomials and the medians of the gamma distributions play a crucial role in the description of $\theta(t)$, as specified in the following.

Theorem 2.2. Let $n \in \mathbb{N}^{*}, \lambda_{n-1}<t \leq \lambda_{n}$ and $a \geq 0$. Then,

$$
\theta(t)-1 \leq \sum_{k=0}^{\infty} C_{k}(t ; n) \frac{(t-a)^{k+1}}{(k+1) !}
$$

if and only if $a \leq \lambda_{n}$. As a consequence,

$$
\theta(t)-1=\sum_{k=0}^{\infty} C_{k}(t ; n) \frac{\left(t-\lambda_{n}\right)^{k+1}}{(k+1) !} .
$$

Setting $t=n$ in (2.5), we obtain formula (1.9). Observe that the coefficients there can be identified in terms of the Charlier polynomials as $c_{k}(n)=C_{k}(n ; n)$. Theorem 2.2 says that we are able to provide upper (respectively, lower) bounds for $\theta(t), t \in\left(\lambda_{n-1}, \lambda_{n}\right]$, if and only if we can give lower (respectively, upper) bounds for $\lambda_{n}$. More precise estimates for $\theta(t)$ are exhibited in the following.

Theorem 2.3. Let $n \in \mathbb{N}^{*}, \lambda_{n-1}<t \leq \lambda_{n}$ and $0 \leq a \leq \lambda_{n} \leq b$. Denote by $d:=\max (|t-a|,|t-b|)$ and assume that $d<1$. Then, we have for any $m \in \mathbb{N}$

$$
\begin{aligned}
& \sum_{k=0}^{2 m} C_{k}(t ; n) \frac{(t-b)^{k+1}}{(k+1) !}-\frac{1}{1-d}\left(\frac{d^{2}}{t}\right)^{m+1} \leq \theta(t)-1 \\
& \leq \sum_{k=0}^{2 m} C_{k}(t ; n) \frac{(t-b)^{k+1}}{(k+1) !}+\frac{1}{1-d}\left(\frac{d^{2}}{t}\right)^{m+1}+(b-a)\left(1+\left(3+\frac{2 d^{2}}{\left(1-d^{2}\right) t}\right) \frac{d}{(1-d) t}\right) .
\end{aligned}
$$

Concerning this result, some remarks are in order. Let $n \in \mathbb{N}^{*}$ and $t \in\left(\lambda_{n-1}, \lambda_{n}\right]$. Observe that $\lambda_{n}=n+O(1)$, as follows from (1.8). Theorem 2.3 gives us exact values of $\theta(t)-1$ up to a remainder term of order $O\left(n^{-m-1}\right)$, whenever we are able to estimate $\lambda_{n}$ in such a way that $b-a=O\left(n^{-m-1}\right)$. The main term of the approximation, that is,

$$
\sum_{k=0}^{2 m} C_{k}(t ; n) \frac{(t-b)^{k+1}}{(k+1) !}
$$

contains in fact terms of order $n^{-k}$ with $k \geq m+1$. However, all of the summands in (2.6) are meaningful, because each one contains terms of order $n^{-k}$ for some $k \leq m$. This will become apparent in the proof of Corollary 2.5 in Section 3 .

As lower and upper estimates of $\lambda_{n}$, we shall consider, respectively, the following candidates:

$$
a_{n}:=n+\frac{2}{3}+\sum_{i=1}^{6} q_{i} n^{-i}
$$


and

$$
b_{n}:=a_{n}+q_{7} n^{-7}
$$

where the rational coefficients $q_{i}, i=1, \ldots, 7$, are given in Table1 1 below. As in Choi [15, such candidates are obtained in the following manner. Start from Marsaglia's expansion of $\theta_{n}$, use formula (2.5) with $t=n$ and equate the coefficients of $n^{-k}$, $k \in \mathbb{N}$, in both sides of this formula, by taking into account the explicit expression given in (2.4).

TABLE 1 . The coefficients $q_{i}, i=1, \ldots, 7$.

\begin{tabular}{cc}
\hline$i$ & $q_{i}$ \\
\hline 1 & $\frac{8}{405}$ \\
2 & $-\frac{64}{5103}$ \\
3 & $\frac{2944}{492075}$ \\
4 & $-\frac{20182528}{15345358875}$ \\
5 & $-\frac{8465604608}{12567848918625}$ \\
6 & $\frac{527611584512}{1696659604014375}$ \\
7 & $\frac{101756461251198976}{136284182692454671875}$ \\
\hline
\end{tabular}

With the preceding notations, we enunciate the following.

Theorem 2.4. For any $n \in \mathbb{N}^{*}$, we have $a_{n}<\lambda_{n}<b_{n}$.

The bounds for $\lambda_{n}$ given in Theorem 2.4 improve those in Choi [15, Theorem 1], Adell and Jodrá [1, Theorem 2] and Alzer [7, Remark 2.8], and therefore complete conjecture 1 by Chen and Rubin [12] stated in (1.8). A consequence of Theorems 2.3 and 2.4 is the following.

Corollary 2.5. Let $u_{n}$ be as in (1.3),$n \in \mathbb{N}^{*}$. Then,

$$
u_{n}-\frac{C_{1}}{n^{7}} \leq \theta_{n} \leq u_{n}+\frac{C_{2}}{n^{7}}
$$

where $C_{1}:=0.01409063087$ and $C_{2}:=0.02193910789$.

The estimates for $\theta_{n}$ in Corollary 2.5 are sharper than the corresponding ones in Choi [15, Theorem 3], Flajolet et al. [18, Theorem 7] and Alm [5, Lemma 10]. Numerical computations show that such estimates are also better than those in (1.5) provided by Alzer [6], whenever $n \geq 3$.

Let $m \in \mathbb{N}^{*}$. Recall that the $m$-th forward difference of a sequence $\left(\alpha_{n}\right)_{n \geq 1}$ is defined by

$$
\Delta_{m} \alpha_{n}:=(A-I)^{m} \alpha_{n}=\sum_{k=0}^{m}(-1)^{m-k}\left(\begin{array}{c}
m \\
k
\end{array}\right) \alpha_{n+k}, \quad n \in \mathbb{N}^{*},
$$

where $A \alpha_{n}:=\alpha_{n+1}, n \in \mathbb{N}^{*}$, and $I$ is the identity operator. The sequence $\left(\alpha_{n}\right)_{n \geq 1}$ is said to be convex of order $m$ if $\Delta_{m} \alpha_{n} \geq 0, n \in \mathbb{N}^{*}$. The inequalities in Theorem 2.4 have the following consequence.

Corollary 2.6. Denote by $\alpha_{n}:=\lambda_{n}-n, n \in \mathbb{N}^{*}$. Then,

$$
(-1)^{m} \Delta_{m} \alpha_{n}>0, \quad m=1, \ldots, 6, \quad n \in \mathbb{N}^{*} .
$$


As it was said in the Introduction, Chen and Rubin [12] conjectured that $\Delta_{1} \alpha_{n} \leq$ $0, n \in \mathbb{N}^{*}$. This has been proved by Alm [5], Adell and Jodrá [1] and Alzer [7]. Observe that the statements in Corollary 2.6 complete Chen and Rubin's conjecture.

\section{THE PROOFS}

Throughout this section, $N_{s}$ stands for a random variable having the Poisson distribution with mean $s$ as in (2.1). For any $s \geq 0$ and $n \in \mathbb{N}$, the well known Poisson-gamma relation states (cf. Johnson et al. [19, p. 164]) that

$$
f_{n}(s):=P\left(N_{s} \leq n\right)=\sum_{k=0}^{n} \frac{e^{-s} s^{k}}{k !}=\int_{s}^{\infty} P\left(N_{u}=n\right) d u=P\left(X_{n}>s\right),
$$

where $X_{n}$ is a random variable having the $\Gamma(n+1,1)$ distribution as in (1.7). From (3.1), it is clear that each function $f_{n}(s)$ is strictly decreasing with $f_{n}(0)=1$ (see Figure 2) and

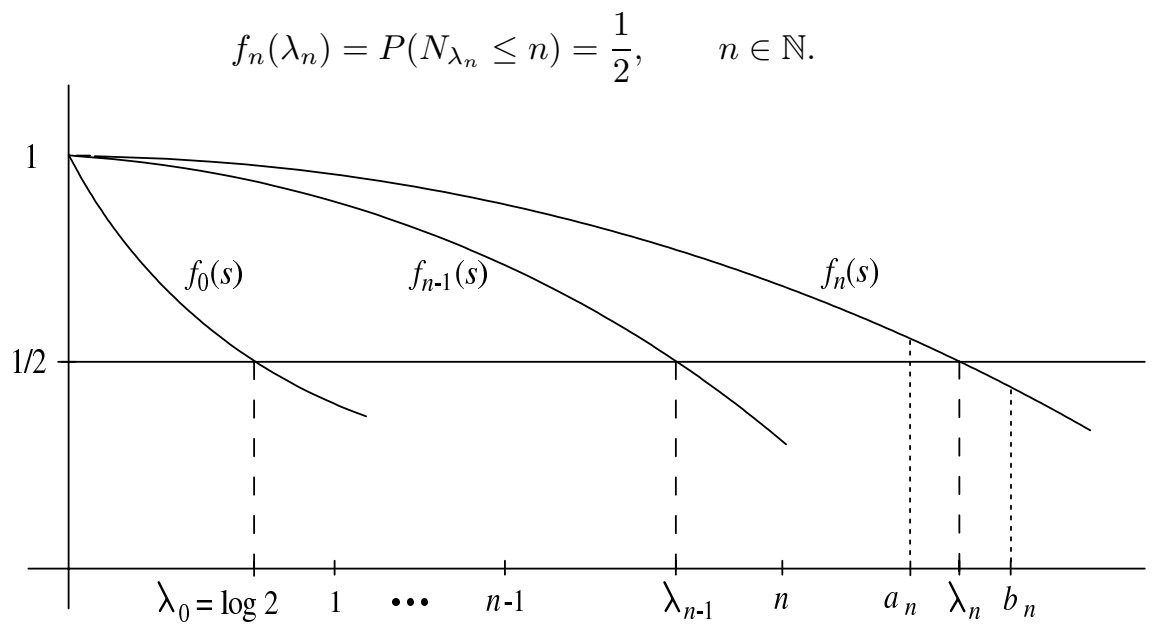

Figure 2. Solutions to $f_{n}(s):=P\left(N_{s} \leq n\right)=1 / 2$.

Proof of Proposition 2.1. Let $n \in \mathbb{N}^{*}$ and $\lambda_{n-1}<t \leq \lambda_{n}$. Multiplying by $e^{-t}$ both sides of (1.11), we obtain the following probabilistic interpretation of $\theta(t)$ :

$$
\frac{1}{2}=P\left(N_{t} \leq n\right)+P\left(N_{t}=n\right)(\theta(t)-1) .
$$

Part (i) readily follows from (3.2) and (3.3) (see Figure 2). To show (ii), it suffices to differentiate in (3.3), by taking into account (3.1) and part (i). The proof is complete.

For any $n \in \mathbb{N}$, we have the asymptotic expansion

$$
P\left(N_{u}=n\right)=P\left(N_{t}=n\right) \sum_{k=0}^{\infty} C_{k}(t ; n) \frac{(t-u)^{k}}{k !}, \quad u \geq 0, \quad t>0 .
$$

Formula (3.4) follows from (2.2) by choosing $x=n$ and $w=u / t-1$. The corresponding finite expansion can be found in Roos [28, Lemma 2 and formula (9)]. See also Adell and Lekuona 4, where (3.4) appears as a particular case of the differential 
calculus for positive linear operators preserving generalized convexity developed in Adell and Lekuona [3].

Proof of Theorem 2.2. Let $n \in \mathbb{N}^{*}$ and $\lambda_{n-1}<t \leq \lambda_{n}$. Assume that $0 \leq a \leq \lambda_{n}$. Using the decrease of $f_{n},(3.2)$ and (3.3), we see that

$$
P\left(N_{a} \leq n\right) \geq P\left(N_{t} \leq n\right)+P\left(N_{t}=n\right)(\theta(t)-1) .
$$

Hence, we obtain from (3.1) and (3.4) that

$$
\theta(t)-1 \leq \frac{1}{P\left(N_{t}=n\right)} \int_{a}^{t} P\left(N_{u}=n\right) d u=\sum_{k=0}^{\infty} C_{k}(t ; n) \frac{(t-a)^{k+1}}{(k+1) !} .
$$

If $a \geq \lambda_{n}$, similar arguments show the converse inequality to (3.5). The proof is complete.

For any real $x$, denote by $\lceil x\rceil$ the ceiling of $x$, that is, the smallest integer not less than $x$. To prove Theorem 2.3. we shall need the following estimates concerning the Chalier polynomials.

Lemma 3.1. Let $n \in \mathbb{N}^{*}, m \in \mathbb{N}$ and $\lambda_{n-1}<t \leq \lambda_{n}$. If $0 \leq d<1$, then

(i) $\sum_{k=2 m+1}^{\infty}\left|C_{k}(t ; n)\right| \frac{d^{k+1}}{(k+1) !} \leq \frac{1}{1-d}\left(\frac{d^{2}}{t}\right)^{m+1}$.

(ii) $\sum_{k=1}^{\infty}\left|C_{k}(t ; n)\right| \frac{d^{k}}{k !} \leq \frac{d}{(1-d) t}\left(3+\frac{2 d^{2}}{\left(1-d^{2}\right) t}\right)$.

Proof. It it is known that $\lambda_{n-1}<n<\lambda_{n}$ and that $\lambda_{n}-\lambda_{n-1}<1$ (see Choi [15, Theorem 1] and Adell and Jodrá [1, Corollary 4]). We therefore have from (2.3)

$$
t\left|C_{k+1}(t ; n)\right| \leq(k+1)\left|C_{k}(t ; n)\right|+k\left|C_{k-1}(t ; n)\right|, \quad k \in \mathbb{N} .
$$

Using induction on $k$ in (3.6), it is seen that

$$
\left|C_{k}(t ; n)\right| \leq(k+1) ! t^{-\lceil k / 2\rceil}, \quad k \in \mathbb{N},
$$

thus implying that

$$
\begin{aligned}
& \sum_{k=2 m+1}^{\infty}\left|C_{k}(t ; n)\right| \frac{d^{k+1}}{(k+1) !} \leq \sum_{k=2 m+1}^{\infty} t^{-\lceil k / 2\rceil} d^{k+1} \\
& =(1+d) \sum_{k=m+1}^{\infty}\left(\frac{d^{2}}{t}\right)^{k} \leq \frac{1}{1-d}\left(\frac{d^{2}}{t}\right)^{m+1} .
\end{aligned}
$$

Similarly, part (ii) follows from (3.7) and a bit of algebra. The proof is complete.

Proof of Theorem 2.3. The lower bound for $\theta(t)-1$ is an immediate consequence of Theorem 2.2 and Lemma3.1(i). On the other hand, it follows from Lemma 3.1(i) that

$$
\begin{aligned}
& \left|\sum_{k=0}^{\infty} C_{k}(t ; n) \frac{(t-a)^{k+1}}{(k+1) !}-\sum_{k=0}^{2 m} C_{k}(t ; n) \frac{(t-b)^{k+1}}{(k+1) !}\right| \\
& \quad \leq \frac{1}{1-d}\left(\frac{d^{2}}{t}\right)^{m+1}+\left|\sum_{k=0}^{2 m} C_{k}(t ; n) \frac{(t-a)^{k+1}-(t-b)^{k+1}}{(k+1) !}\right| .
\end{aligned}
$$


Using the inequality

$$
y^{k+1}-x^{k+1} \leq(k+1)(y-x) y^{k}, \quad 0 \leq x \leq y, \quad k \in \mathbb{N},
$$

in the second term on the right-hand side in (3.8), the upper bound for $\theta(t)-1$ follows from Theorem 2.2 and Lemma 3.1(ii). This completes the proof.

Let $\left(t_{n}\right)_{n \geq 1}$ be a sequence such that $t_{n} \in[n, n+1], n \in \mathbb{N}^{*}$. For any $k \in \mathbb{N}$ and $n \in \mathbb{N}^{*}$, we denote it by

$$
\begin{aligned}
D_{k}\left(t_{n}\right):= & C_{k}(n+1 ; n+1) \\
& \times\left(\frac{\left(n+1-t_{n}\right)^{k+1}-\left(n+1-t_{n+1}\right)^{k+1}}{(k+1) !}-\frac{\left(n+1-t_{n}\right)^{k}}{k !}\right) .
\end{aligned}
$$

The following auxiliary result, which is interesting by itself, provides a criterion to give upper and lower bounds for $\lambda_{n}$ by checking the monotonicity of certain sequences involving tail probabilities of appropriate Poisson distributions. In fact, statement (ii) in Lemma 3.2 below is connected with the property of monotonic convergence in the central limit theorem. In this respect, suppose we are given a stochastic process $\left(X_{s}\right)_{s>0}$ obeying the central limit theorem, that is, verifying that

$$
\lim _{s \rightarrow \infty} P\left(X_{s} \leq x\right)=P(Z \leq x), \quad x \in \mathbb{R},
$$

where $Z$ is a standard normal random variable. In many cases, it is interesting to know if the convergence in (3.11) is monotonic for certain values of $x$ (see Teicher [30] and Kane [20]). On the other hand, it is well known that the standardized Poisson process $\left(\left(N_{s}-s\right) / \sqrt{s}\right)_{s>0}$ obeys the central limit theorem. Therefore, if $t_{n} \in[n, n+1]$ for any $n \in \mathbb{N}^{*}$, then

$$
\lim _{n \rightarrow \infty} P\left(N_{t_{n}} \leq n\right)=\lim _{n \rightarrow \infty} P\left(\frac{N_{t_{n}}-t_{n}}{\sqrt{t_{n}}} \leq \frac{n-t_{n}}{\sqrt{t_{n}}}\right)=P(Z \leq 0)=1 / 2 .
$$

Lemma 3.2 gives necessary and sufficient conditions to ensure that the sequence $\left(P\left(N_{t_{n}} \leq n\right)\right)_{n \geq 1}$ strictly decreases. Let $a_{n}$ and $b_{n}$ be as in (2.7) and (2.8), respectively. It turns out that the sequence $\left(P\left(N_{a_{n}} \leq n\right)\right)_{n \geq 1}$ strictly decreases, whereas the sequence $\left(P\left(N_{b_{n}} \leq n\right)\right)_{n \geq 1}$ strictly increases (see (3.14), (3.17) and (3.18) below). Since $b_{n}=a_{n}+O\left(n^{-7}\right)$, the preceding statements suggest that the property of monotonic convergence in the central limit theorem is a very delicate question (see Kane 20] for further evidence).

Lemma 3.2. Let $\left(t_{n}\right)_{n \geq 1}$ be a sequence such that $t_{n} \in[n, n+1], n \in \mathbb{N}^{*}$. For each fixed $n \in \mathbb{N}^{*}$, the following assertions are equivalent:

(i) $\sum_{k=0}^{\infty} D_{k}\left(t_{n}\right)>0$.

(ii) $P\left(N_{t_{n}} \leq n\right)>P\left(N_{t_{n+1}} \leq n+1\right)$.

If, in addition, either (i) or (ii) are satisfied for any $n \in \mathbb{N}^{*}$, then

$$
\lambda_{n}>t_{n}, \quad n \in \mathbb{N}^{*} .
$$

All of the preceding statements remain valid if we replace ' $>$ ' by ' $<$ '. 
Proof. Let $n \in \mathbb{N}^{*}$ be fixed. By (3.1) and (3.4), we have

$$
\begin{aligned}
& P\left(N_{t_{n}} \leq n\right)-P\left(N_{t_{n+1}} \leq n+1\right)=\int_{t_{n}}^{t_{n+1}} P\left(N_{u}=n+1\right) d u-P\left(N_{t_{n}}=n+1\right) \\
& =P\left(N_{n+1}=n+1\right) \sum_{k=0}^{\infty} \frac{C_{k}(n+1 ; n+1)}{k !}\left(\int_{t_{n}}^{t_{n+1}}(n+1-u)^{k} d u-\left(n+1-t_{n}\right)^{k}\right) \\
& =P\left(N_{n+1}=n+1\right) \sum_{k=0}^{\infty} D_{k}\left(t_{n}\right) .
\end{aligned}
$$

This shows the equivalence between (i) and (ii). Assume that (ii) is satisfied for any $n \in \mathbb{N}^{*}$. By (3.12), the sequence $\left(P\left(N_{t_{n}} \leq n\right)\right)_{n>1}$ decreasingly converges to $1 / 2$ as $n \rightarrow \infty$, thus implying that $P\left(N_{t_{n}} \leq n\right)>1 / 2=P\left(N_{\lambda_{n}} \leq n\right), n \in \mathbb{N}^{*}$, as follows from (3.2). From the decrease of $f_{n}(s)$ (see Figure 2), we conclude that $\lambda_{n}>t_{n}, n \in \mathbb{N}^{*}$. This completes the proof.

Before proving Theorem 2.4, we give the estimates

$$
\sum_{k=2 m+1}^{\infty}\left|C_{k}(n+1 ; n+1)\right| \frac{d^{k}}{k !} \leq \frac{1}{d(1-d)}\left(\frac{d^{2}}{n+1}\right)^{m+1}, \quad m, n \in \mathbb{N},
$$

where $d \in[0,1)$. Such inequalities are shown in a similar way as those in Lemma 3.1(i).

Proof of Theorem 2.4. Let us show the lower bound. Let $n \in \mathbb{N}^{*}$ and let $a_{n}$ be as in (2.7). We first claim that

$$
\sum_{k=0}^{\infty} D_{k}\left(a_{n}\right)>0, \quad n \geq 21
$$

where $D_{k}\left(a_{n}\right)$ is defined in (3.10). Indeed, let $n \geq 21$ and denote it by $d:=$ $2 / 3+1 / 75$. Since $a_{n+1}-a_{n}<1, a_{n+1}-(n+1)<d$ and $n+1-a_{n} \leq 1 / 3$, we have from (3.9) and (3.10)

$$
\left|D_{k}\left(a_{n}\right)\right|<\left|C_{k}(n+1 ; n+1)\right| \frac{d^{k}+(1 / 3)^{k}}{k !}, \quad k \in \mathbb{N} .
$$

By virtue of (3.13), this implies that

$$
\sum_{k=17}^{\infty}\left|D_{k}\left(a_{n}\right)\right|<\left(\frac{d^{17}}{1-d}+\frac{(1 / 3)^{17}}{1-1 / 3}\right) \frac{1}{(n+1)^{9}} .
$$

On the other hand, disregarding the positive terms of order $n^{-k}, k \geq 10$, on the left-hand side in (3.16), and bounding from below all of the negative terms of order $n^{-k}, k \geq 88$, by $-2.15 \cdot 10^{25} n^{29}(n+1)^{-117}$, we obtain

$$
\begin{aligned}
\sum_{k=0}^{16} D_{k}\left(a_{n}\right)> & \frac{50878230625599488 n^{109}}{9085612179496978125(1+n)^{117}} \\
& +\frac{4951728238843765105229 n^{108}}{7880753114940625920000(1+n)^{117}}-\frac{2.15 \cdot 10^{25} n^{29}}{(n+1)^{117}}
\end{aligned}
$$


This, together with (3.15), shows claim (3.14). Finally, it follows from Table 2 below that $P\left(N_{a_{n}} \leq n\right)>P\left(N_{a_{n+1}} \leq n+1\right), n=1, \ldots, 20$. By Lemma 3.2. this means that

$$
\sum_{k=0}^{\infty} D_{k}\left(a_{n}\right)>0, \quad n=1, \ldots, 20 .
$$

In view of Lemma 3.2, the lower bound in Theorem 2.4 follows from (3.14) and (3.17).

The proof of the upper bound is analogous, since it is based on the fact that

$$
\sum_{k=0}^{\infty} D_{k}\left(b_{n}\right)<0, \quad n \in \mathbb{N}^{*}
$$

where $b_{n}$ is defined in (2.8). Details are omitted.

TABLE 2. Values of $P\left(N_{a_{n}} \leq n\right)-P\left(N_{a_{n+1}} \leq n+1\right)$ for small $n$ computed by Maple with 25 digits' precision.

\begin{tabular}{llll}
\hline$n$ & $P\left(N_{a_{n}} \leq n\right)-P\left(N_{a_{n+1}} \leq n+1\right)$ & $n$ & $P\left(N_{a_{n}} \leq n\right)-P\left(N_{a_{n+1}} \leq n+1\right)$ \\
\hline 1 & $0.505994160732982535966 \cdot 10^{-4}$ & 11 & $0.19710118062552 \cdot 10^{-11}$ \\
2 & $0.7041131171423239324 \cdot 10^{-6}$ & 12 & $0.9764882154887 \cdot 10^{-12}$ \\
3 & $0.422552667758349646 \cdot 10^{-7}$ & 13 & $0.5104741082694 \cdot 10^{-12}$ \\
4 & $0.51710313695231388 \cdot 10^{-8}$ & 14 & $0.2794399295429 \cdot 10^{-12}$ \\
5 & $0.9646813708129802 \cdot 10^{-9}$ & 15 & $0.1592037635986 \cdot 10^{-12}$ \\
6 & $0.2380036710511234 \cdot 10^{-9}$ & 16 & $0.939307344937 \cdot 10^{-13}$ \\
7 & $0.716647097487652 \cdot 10^{-10}$ & 17 & $0.571572615703 \cdot 10^{-13}$ \\
8 & $0.250539373485964 \cdot 10^{-10}$ & 18 & $0.357486921311 \cdot 10^{-13}$ \\
9 & $0.98375505857939 \cdot 10^{-11}$ & 19 & $0.229151884312 \cdot 10^{-13}$ \\
10 & $0.42390196405984 \cdot 10^{-11}$ & 20 & $0.150175050868 \cdot 10^{-13}$ \\
\hline
\end{tabular}

Proof of Corollary 2.5. We shall only show the upper bound, because the proof of the lower bound follows a similar pattern. Let $n \in \mathbb{N}^{*}$ and let $u_{n}$ be as in (1.3). Looking at Theorem [2.3, we set $t=n, m=6, a=a_{n}$ and $b=b_{n}$, as defined in (2.7) and (2.8), respectively. Observe that $\max \left(a_{n}-n, b_{n}-n\right) \leq d:=2 / 3+1 / 75$. Therefore, applying Theorem 2.3 and bounding above all of the positive terms of order $n^{-k}, k=8,9$, as $c n^{-k} \leq c n^{-7}$, we arrive at

$$
\theta_{n} \leq 1+\sum_{k=0}^{12} C_{k}(n ; n) \frac{\left(n-b_{n}\right)^{k+1}}{(k+1) !}+\frac{C^{*}}{n^{7}},
$$

where $C^{*}:=0.02236047308$. Disregarding the negative terms of order $n^{-k}, k \geq 8$, on the left-hand side in (3.20), and bounding above all of the positive terms of order $n^{-k}, k \geq 7$, as $c n^{-k} \leq c n^{-7}$, we obtain

$$
1+\sum_{k=0}^{12} C_{k}(n ; n) \frac{\left(n-b_{n}\right)^{k+1}}{(k+1) !} \leq u_{n}-\frac{\widetilde{C}}{n^{7}},
$$


where $\widetilde{C}:=0.0004213651893$. Thus, the upper bound in Corollary 2.5 follows from (3.19) and (3.20). The proof is complete.

Proof of Corollary 2.6. We shall only show that $\Delta_{6} \alpha_{n}>0, n \in \mathbb{N}^{*}$. Recalling (2.7) -(2.9) and Theorem 2.4, we have

$$
\begin{aligned}
& \Delta_{6} \alpha_{n}=\sum_{k=0}^{6}(-1)^{6-k}\left(\begin{array}{l}
6 \\
k
\end{array}\right) \lambda_{n+k} \\
& >a_{n}-6 b_{n+1}+15 a_{n+2}-20 b_{n+3}+15 a_{n+4}-6 b_{n+5}+a_{n+6} \\
& =\sum_{i=1}^{6} q_{i} r_{i}(n)-q_{7}\left(\frac{6}{(n+1)^{7}}+\frac{20}{(n+3)^{7}}+\frac{6}{(n+5)^{7}}\right)>0
\end{aligned}
$$

where

$$
r_{i}(n):=\sum_{k=0}^{6}(-1)^{6-k}\left(\begin{array}{l}
6 \\
k
\end{array}\right)(n+k)^{-i}, \quad i=1, \ldots, 6,
$$

and the coefficients $q_{i}, i=1, \ldots, 7$, are those given in Table 1 . This concludes the proof.

Remark 3.3. The necessary symbolic and numerical computations in this paper have been performed with the help of the computer algebra system Maple, Release 9.01. Particularly, in the proofs of Theorem 2.4 and Corollary 2.5. Maple yields a fraction decomposition of the finite sums on the left-hand sides in (3.16) and (3.20) which leads, together with (2.4), to the estimates on their corresponding right-hand sides.

\section{OpEN PROBLEMS}

The statements in Theorem 2.4 and Corollaries 2.5 and 2.6 may be improved by considering more exact terms in the asymptotic expansions of $\theta_{n}$ and $\lambda_{n}$. In view of the methodology developed in this paper, the only limitation for an arbitrary improvement is to compute efficiently, with the help of symbolic calculus programs, all of the finite sums we need. In particular, Corollary 2.6 can be strengthened as

$$
(-1)^{m} \Delta_{m} \alpha_{n}>0, \quad m=1, \ldots, 14, \quad n \in \mathbb{N}^{*} .
$$

In this regard, recall that a function $f$ defined on $\mathbb{N}$ is said to be completely monotone if $(-1)^{m} \Delta_{m} f(n) \geq 0, m, n \in \mathbb{N}$, where it is understood that $\Delta_{0} f:=f$. The celebrated Hausdorff's characterization criterion states (see, for instance, Feller [17. p. 225]) that a completely monotone function $f$ with $f(0)=1$ coincides with the moment sequence of a unique probability distribution function. In other words, there exists a unique random variable $X$ taking on values in $[0,1]$, with distribution function $F$, such that

$$
f(n)=\int_{0}^{1} x^{n} d F(x), \quad n \in \mathbb{N} .
$$

Recently, the authors have shown in [2] that the sequence $\left(\theta_{n}\right)_{n \geq 0}$ is completely monotone. On the other hand, Alzer [7, Remark 3.4] has conjectured that $\left(\alpha_{n}\right)_{n \geq 0}$ fulfills the same property. The inequalities in (4.1) strongly suggest that Alzer's conjecture is true. If this was the case, the problem would be to determine the associated random variable whose distribution function satisfies the moment equalities given in (4.2). Finally, let $\lambda(t)$ be the median of the $\Gamma(t+1,1)$ distribution, 
$t>-1$, and denote by $\lambda^{(k)}(t)$ the $k$-th derivative of $\lambda(t)$. Berg and Pedersen 8 have shown that $(-1)^{k} \lambda^{(k)}(t)>0$ for sufficiently large values of $t$ and $k=2,3, \ldots$. This again reinforces Alzer's conjecture.

\section{REFERENCES}

1. J. A. Adell and P. Jodrá, Sharp estimates for the median of the $\Gamma(n+1,1)$ distribution, Statist. Probab. Lett. 71 (2005), 185-191. MR2126774 (2006b:60027)

2. J. A. Adell and P. Jodrá, On the complete monotonicity of a Ramanujan sequence connected with $e^{n}$, to appear in The Ramanujan Journal.

3. J. A. Adell and A. Lekuona, Taylor's formula and preservation of generalized convexity for positive linear operators, J. Appl. Prob. 37 (2000), 765-777. MR1782452 (2001e:41031)

4. J. A. Adell and A. Lekuona, Sharp estimates in signed Poisson approximation of Poisson mixtures, Bernoulli 11 (2005), 47-65. MR2121455 (2005k:62051)

5. S. E. Alm, Monotonicity of the difference between median and mean of gamma distributions and of a related Ramanujan sequence, Bernoulli 9 (2003), 351-371. MR.1997033(2004e:60024)

6. H. Alzer, On Ramanujan's inequalities for $\exp (k)$, J. London Math. Soc. (2) 69 (2004), 639656. MR2050038(2006c:33001)

7. H. Alzer, Proof of the Chen-Rubin conjecture, Proc. Roy. Soc. Edinburgh Sect. A 135 (2005), 677-688. MR2173334 (2007a:62017)

8. C. Berg and H. L. Pedersen, The Chen-Rubin conjecture in a continuous setting. Preprint.

9. B. C. Berndt, Ramanujan's Notebooks, Part II, Springer-Verlag, New York, 1989. MR970033 (90b:01039)

10. B. C. Berndt, Y.-S. Choi and S.-Y. Kang, The problems submitted by Ramanujan to the Journal of the Indian Mathematical Society, Continued Fractions: From Analytic Number Theory to Constructive Approximation (Columbia, MO, 1998), Contem. Math. 236 (American Mathematical Society, Providence, RI, 1999), 15-56. MR 1665361 (2000i:11003)

11. P. Bracken, A function related to the central limit theorem, Comment. Math. Univ. Carolin. 39 (1998), 765-775. MR1715465 (2001a:33002)

12. J. Chen and H. Rubin, Bounds for the difference between median and mean of Gamma and Poisson distributions, Statist. Probab. Lett. 4 (1986), 281-283. MR858317 (88a:60040)

13. T. T. Cheng, The normal approximation to the Poisson distribution and a proof of a conjecture of Ramanujan, Bull. Amer. Math. Soc. 55 (1949), 396-401. MR0029487 (10:613d)

14. T. S. Chihara, An Introduction to Orthogonal Polynomials, Gordon and Breach, New York, 1978. MR0481884 (58:1979)

15. K. P. Choi, On the medians of gamma distributions and an equation of Ramanujan, Proc. Amer. Math. Soc. 121 (1994), 245-251. MR1195477 (94g:62025)

16. E. T. Copson, An approximation connected with $e^{-x}$, Proc. Edinburgh Math. Soc. (2) 3 (1933), 201-206.

17. W. Feller, An Introduction to Probability Theory and Its Applications, Vol. II, John Wiley \& Sons, New York, 1966. MR0210154 (35:1048)

18. P. Flajolet, P. J. Grabner, P. Kirschenhofer and H. Prodinger, On Ramanujan's $Q$-function, J. Comput. Appl. Math. 58 (1995), 103-116. MR.1344359(96f:65010)

19. N. L. Johnson, S. Kotz and A. W. Kemp, Univariate Discrete Distributions, 2nd edition, Wiley, New York, 1993. MR1224449 (95d:62018)

20. J. M. Kane, Monotonic approach to central limits, Proc. Amer. Math. Soc. 129 (2001), 21272133. MR $1825926(2002 \mathrm{~b}: 60035)$

21. J. Karamata, Sur quelques problèmes posés par Ramanujan, J. Indian Math. Soc. 24 (1960), 343-365. MR0133307 (24:A3141)

22. E. S. Key, A probabilistic approach to a conjecture of Ramanujan, J. Ramanujan Math. Soc. 4 (1989), 109-119. MR.1040203 (91d:11100)

23. J. C. W. Marsaglia, The incomplete Gamma function and Ramanujan's rational approximation to $e^{x}$, J. Statis. Comput. Simul. 24 (1986), 163-169.

24. R. B. Paris, On a generalisation of a result of Ramanujan connected with the exponential series, Proc. Edinburgh Math. Soc. (2) 24 (1981), 179-195. MR633719 (83b:33004)

25. S. Ramanujan, Question 294, J. Indian Math. Soc. 3 (1911), 128.

26. S. Ramanujan, On question 294, J. Indian Math. Soc. 4 (1912), 151-152.

27. S. Ramanujan, Collected Papers, Chelsea, New York, 1962. 
28. B. Roos, Improvements in the Poisson approximation of mixed Poisson distributions, J. Statist. Plann. Inference 113 (2003), 467-483. MR.1965122 (2004e:60043)

29. G. Szegö, Über einige von S. Ramanujan gestelle Aufgaben, J. London Math. Soc. 3 (1928), $225-232$.

30. H. Teicher, An inequality on Poisson probabilities, Ann. Math. Statist. 26 (1955), 147-149. MR0067384 (16:722f)

31. G. N. Watson, Theorems stated by Ramanujan (V): approximations connected with $e^{x}$, Proc. London Math. Soc. 29 (1929), 293-308.

Departamento de Métodos Estadísticos, Universidad de Zaragoza, 50009 Zaragoza, SPAIN

E-mail address: adell@unizar.es

Departamento de Métodos Estadísticos, Universidad de Zaragoza, 50009 Zaragoza, SPAIN

E-mail address: pjodra@unizar.es 